\title{
Simulations and Measurements of Large Phase Progression in Multi-layered Metamaterials
}

\author{
Amir I. Zaghloul*, Steven J. Weiss, and Theodore K. Anthony \\ US Army Research Laboratory, Adelphi, MD 20783, USA \\ amir.i.zaghloul.civ@mail.mil
}

\begin{abstract}
Insertion phase in multi-layered metamaterials play an important role in understanding the constitutive parameters of the material. Simulations and measurements show the distinct large phase change across the frequency band in which the metamaterial exhibits special parameters. This paper addresses the phase behavior for materials that use loops or rings for magnetic properties and rods, wires, or probes for electric properties. Continuous wires and cut wires in the structure show different properties, especially in the frequency regions where negative refraction occurs. The conjecture that negative refraction is a manifestation of insertion phase variations is reiterated in this paper.
\end{abstract}

\section{INTRODUCTION}

In characterizing the constitutive parameters of materials, the scattering matrix parameters are often used as a starting point. This has been done extensively for metamaterials [1]. Some of the derivations use expressions that calculate the insertion phase in the medium in a modular $2 \pi$, or wrapped values. Depending on the parameters of the medium, this may result in refraction of the transmitted beam in a direction that may be interpreted as a negative refraction. Alternatively, if true unwrapped values of the propagation phase are used, the negative refraction appearance may be interpreted as a grating-lobe like effect in arrays of narrow element spacing with large phase taper between the elements [2], [3]. Measurements, manipulated to show the unwrapped large phase propagation for layered metamaterials were reported in [4], and showed how the phase variation builds up in consecutive layers in the material. Although this was shown for ring resonator and loops types of metamaterials, it may be possible to show it in the circuit-component-based metamaterials [5].

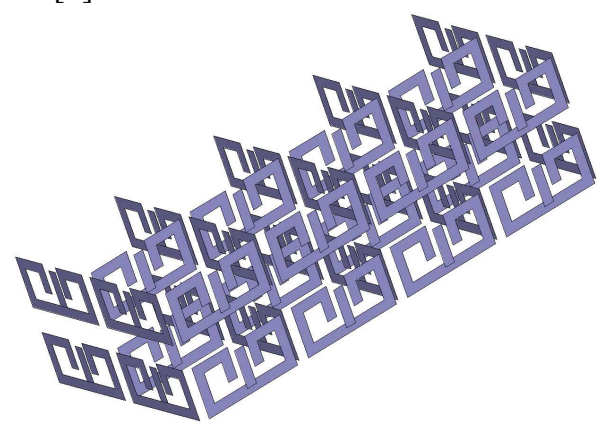

Fig. 1: Multi-layered metamaterial that used capacitivelyloaded loops plus probes (CLL-P) as the basic element
In this paper, we simulate the phase propagation across multilayered metamaterials to show the large phase variation within narrow frequency bands. The simulation tool, HFSS in this case, is manipulated to calculate the unwrapped phase, instead of the $2 \pi$-modular phase. The metamaterial selected for this analysis is a capacitively loaded loop (CLL) on one side of a substrate with a probe or wire on the opposite side, as shown in Figure 1. The incident plane is polarized in the direction of the probe (or wire) in the metamaterial, i.e. vertical. Some of the results show a cross-polarized field that is perpendicular to the wires, or horizontal. Measurement results are also given for the same structure, but with different propagation arrangement. Both simulation and measurements show large propagation phase through the metamaterial.

\section{Simulation OF THE CUT-WIRE AND CONTINUOUS-WIRE CONFIGURATIONS}

The extent of the probe or wire in the unit cell can be based on a cut-wire or continuous-wire arrangement. In the cut-wire scenario, the probe length on the other side of the loop is limited to the loop height. In the continuous wire scenario, the wire continues, covering the gaps between loops. A plane wave is assumed to be incident on a four-layer metamaterial, which extends indefinitely in the perpendicular plane.

Although it was reported that the negative refraction occurs in the continuous wire case, the propagation phase is large within certain bandwidth in both cases. Figure 2 shows the propagation phase for the cut-wire design, as compared with free space propagation. An upward large phase variation is noticed over a bandwidth before it settles down to a regular material slope.

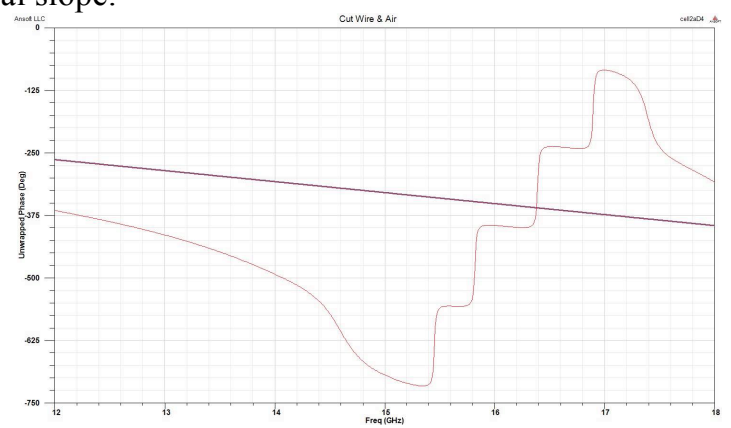

Fig. 2: Propagation phase in free space and in a cut-wire arrangement showing a bandwidth over which the phase variation is large and slopes upward 
Figure 3 shows the propagation phase variation versus frequency for the continuous wire arrangement. In this case, the phase variation is steep with a downward slope over a narrow bandwidth before it slows down to the low-phase variation of regular materials.

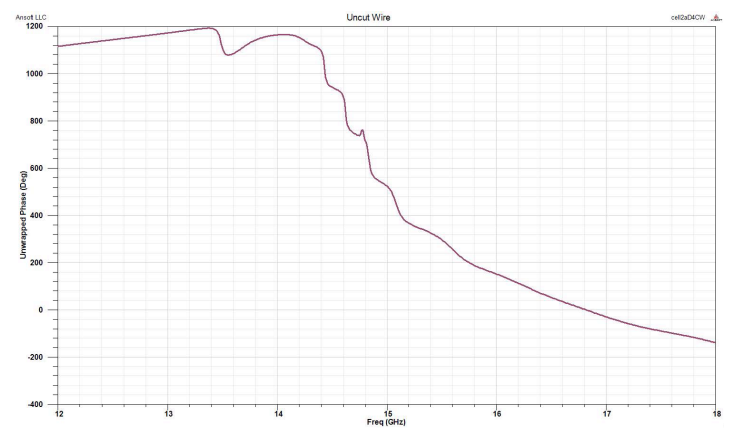

Fig. 3: Propagation phase in a continuous-wire arrangement showing a narrow bandwidth over which the phase variation is large and slopes downward

The $\mathrm{S}$ parameters of the propagation through the CLL-P material show similar bandwidth behaviour as the propagation phase. Figure 4 is a plot of the insertion loss and return loss for the cut-wire case. The large bandwidth over which almost complete reflection occurs matches the bandwidth over which the propagation phase is high, which is distinctly different than the more benign behaviour across most of the band. Figure 5 shows the different behaviour of the continuous-wire material. Here the sharp phase variation bandwidth corresponds to a much lower insertion loss than the cut-wire case. This is the region in which the continuous-wire arrangement shows negative refractive index. The steepest phase variation over this band supports the conjecture that the negative refraction is a result of a grating-lobe-like behaviour that appears as a refraction in a direction away from the main plane wave direction [2], [3].

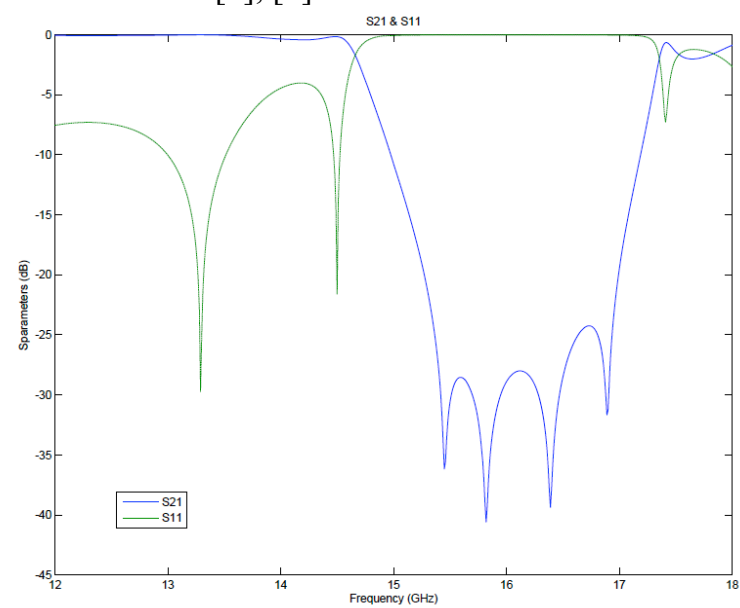

Fig. 4: Insertion (blue) and return (green) loss for the cutwire arrangement showing a bandwidth similar to the phase variation bandwidth

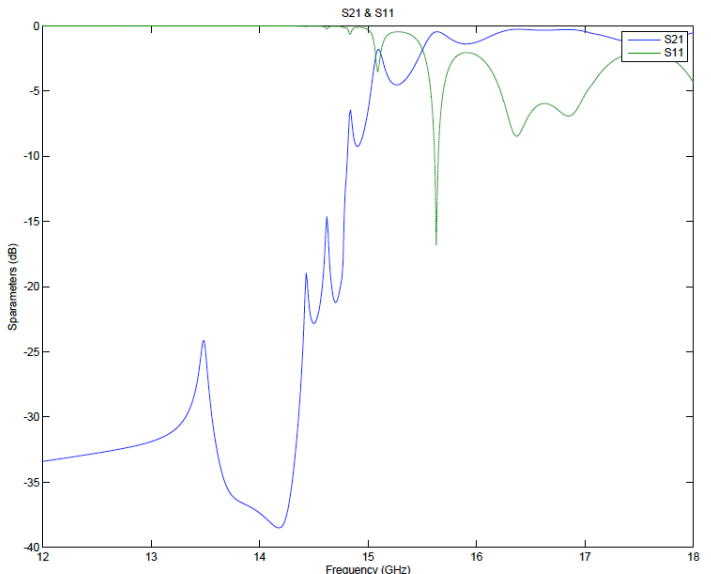

Fig. 5: Insertion (blue) and return (green) loss for the continuous-wire arrangement showing a bandwidth similar to the phase variation bandwidth

\section{MEASUREMENT RESULTS OF A 4-LAYER CLL-P METAMATERIAL BLOCK}

To verify the large propagation phase in the metamaterial, a 4-layer CLL-P structure of finite planar dimensions was fabricated and tested. In this case only the cut-wire arrangement is implemented. The measurement set up was different than the simulated one. Because of the finiteness of the plane that is repeated over the four layers, the transmitting and receiving ports have to be close to the material block in order to avoid diffraction components from around the block. The transmitting and receiving ports are aligned waveguide ports. As such, the simulated plane-wave arrangement is considerably different than the measurement arrangement. This resulted in a shift in the frequency band over which the material acts as a metamaterial. Figure 6 shows the measured phase change over the material path as compared to the freespace phase response. The sharp drop in phase looks similar to the simulated continuous-wire case. It can be argued that because the small spacing between the rows or planes in the structure, strong coupling may occur between the wires in different planes, hence the continuous-wire-like behaviour.

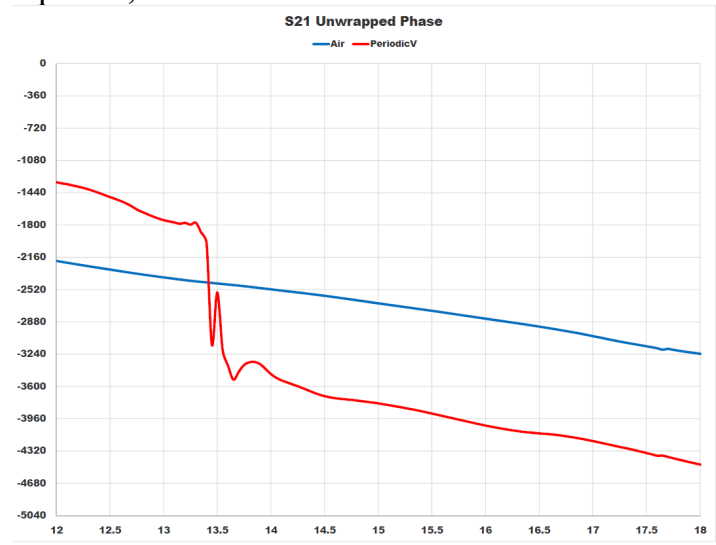

Fig. 6: Measured propagation phase in free space (blue) and in a 4-layer CLL-P material showing a narrow bandwidth over which the phase variation is large and slopes downward 
The measured insertion and return losses are shown in Figure 7. As in the simulation, the correlation in the frequency band between the large insertion phase slope and the distinct insertion loss and return loss behaviours is apparent. The figure shows the measured free space parameters for comparison.

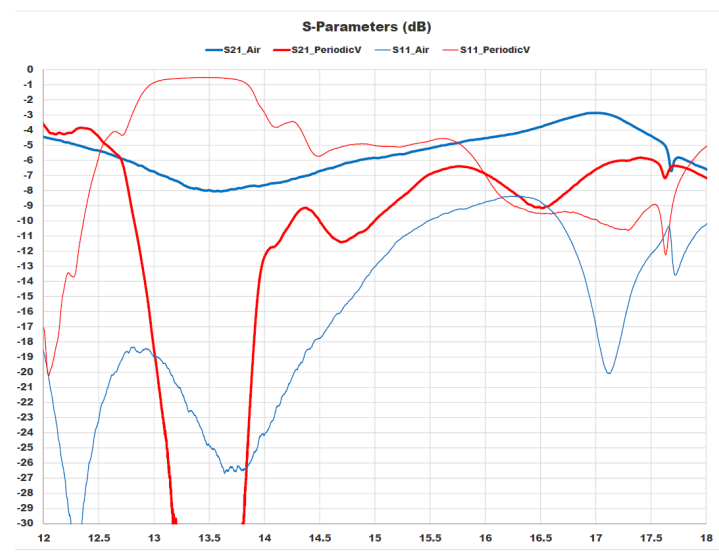

Fig. 7: Measured insertion (bold red) and return (light red) loss for a 4-layer CLL-P material showing a bandwidth close to the phase variation bandwidth as compared to the freespace response (bold and light blue)

\section{CONCLUSION}

Metamaterial structures exhibit large insertion phase variations with steep slopes versus frequency within narrow segments of the frequency band. The large phase variation, which is observed only when the unwrapped phase is simulated or measured, is associated with corresponding special insertion loss and return losses in the material. The same frequency band also corresponds to distinctly special values of the material's constitutive parameters, which may be negative. The constitutive parameters lead to refractive index values that may be negative as well. The large phase variations within the narrow frequency band create a gratinglobe like condition that causes the effect of negative refraction in arrays of narrowly spaced elements.

\section{REFERENCES}

[1] D.R. Smith, D.C. Vier, Th. Koschny, and C.M. Soukoulis," Electromagnetic Parameter Retrieval from Inhomogeneous Metamaterials," Physical Review E 71, 2005.

[2] G. Talalai, S. Weiss, T. Garner, and A. Zaghloul, "Relationship between Phased Arrays and Negative Refraction," ACES Conference, Williamsburg, VA, March 2015.

[3] S.J. Weiss, G. Talalai, and A. Zaghloul, "Negative Refraction and Array Theory," Antenna Applications Symposium at Allerton, Illinois, September 2015.

[4] A.I. Zaghloul, T.K. Anthony, S.J. Weiss, and G. Talalai, "Measurements and Significance of Phase Progression in Metamaterials," Antenna Applications Symposium at Allerton, Illinois, September 2015.

[5] A.K. Ayer, P.K. Kremer, and G.V. Eleftheriades, "Experimental and Theoretical Verification of Focusing in a Large, Periodically Loaded Transmission Line Negative Refractive Index Metamaterial," Optical Express, Vol. 11, No. 7, April 2003. 